\title{
Alimentação saudável na perspectiva multiprofissional: A experiência do Projeto "Saúde no Prato" desenvolvido na Atenção Básica
}

\section{Healthy nutrition in the multiprofessional perspective: The experience of the "Health on the Plate" Project developed in Primary Care}

\author{
Arianne Albuquerque Santos Almeida \\ Hospital Universitário Professor Alberto Antunes, Residência Multiprofissional em Saúde do Adulto e do Idoso. Avenida Lourival Melo Mota, s/n - \\ Tabuleiro dos Martins, Maceió, Alagoas, 57072-900. Fone: (82) 3202-3800. E-mail: ariannealbuquerque26@gmail.com \\ Sonaly Costa Silva \\ Hospital Universitário Professor Alberto Antunes, Residência Multiprofissional em Saúde do Adulto e do Idoso. Tabuleiro dos Martins, Maceió, \\ Alagoas. Fone: (82) 3202-3800. E-mail: sonalycs@gmail.com \\ Werle Thayane Costa de Araújo \\ Hospital Universitário Professor Alberto Antunes, Residência Multiprofissional em Saúde do Adulto e do Idoso. Tabuleiro dos Martins, Maceió, \\ Alagoas. Fone: (82) 3202-3800. E-mail: werlethayane@ hotmail.com \\ Sandra Mary Lima Vasconcelos \\ Hospital Universitário Professor Alberto Antunes, Residência Multiprofissional em Saúde do Adulto e do Idoso. Maceió, Alagoas. Fone: (82) 3202 \\ 3800. Universidade Federal de Alagoas, Faculdade de Nutrição. Laboratório de Nutrição em Cardiologia. Tabuleiro dos Martins. Maceió, Alagoas. \\ Fone: (82) 3214-1160. E-mail: sandra-mary@hotmail.com \\ Maria Tereza Soares de Mélo \\ Unidade Básica de Saúde Graciliano Ramos. Maceió, Alagoas. Fone: (82) 3315-5441. E-mail: mtecamelo@yahoo.com.br.
}

Resumo: O projeto denominado "Saúde no Prato" teve inicio no primeiro semestre do ano de 2016. Portanto, objetivou-se com este relato descrever a experiência multiprofissional nas atividades de educação alimentar e nutricional e contribuir para a disseminação de experiências relacionadas a este modelo de intervenção na Atenção Básica. Trata-se de um estudo descritivo do tipo relato de experiência acerca da implantação de um projeto desenvolvido por residentes de Nutrição, Enfermagem, Serviço Social, Psicologia e Farmácia do programa de Residência Multiprofissional em Saúde do Adulto e Idoso do Hospital Universitário Professor Alberto Antunes da Universidade Federal de Alagoas, e com profissionais de saúde de uma UBS, onde é realizado o estágio em Atenção Básica da residência. O projeto foi desenvolvido em encontros com os profissionais e usuários inscritos, totalizando seis encontros semanais cujo formato padrão incluía a exposição do tema seguida de uma oficina culinária. Foi possível observar o sucesso do projeto ao longo dos encontros, pois as pessoas participaram de forma ativa, trazendo um feedback positivo através das mudanças de hábitos alimentares, mostrando que a educação nutricional em grupo constitui uma estratégia de fundamental importância para o enfrentamento dos problemas alimentares e nutricionais. Somados a esses pontos positivos, foram citados ainda pelos participantes: redução do peso, da pressão arterial, mudanças de práticas alimentares na família (redução do consumo de sal, açúcar, alimentos industrializados e aumento no consumo de saladas e frutas), maior cuidado na preparação dos alimentos, confecção de horta vertical em casa, maior participação dos homens na cozinha e maior dedicação às caminhadas.

Palavras-chave: Educação alimentar e nutricional; Atenção primária à saúde; Prática de grupo.

Abstract: The project entitled "Health on the Prato" started in the first half of 2016. The main objective of this report is to describe the multiprofessional experience in food and nutritional education activities and to contribute to the dissemination of experiences related to this intervention model in Attention Basic. This is a descriptive study of the type of experience related to the implementation of a project designed by residents of Nutrition, Nursing, Social Work, Psychology and Pharmacy of the Residency program Multiprofessional in Adult and Elderly Health at the Professor Alberto Antunes University Hospital of the Federal University of Alagoas, and with health professionals from a UBS, where the internship is carried out in basic care of the residence. The project was developed in meetings with professionals and registered users, totaling six weekly meetings, where in each meeting the standard format included the exposition of the theme followed by a cooking workshop. It was possible to observe the success of the project throughout the meetings, as people actively participated, bringing positive feedback through changes in eating habits showing that group nutrition education is a strategy of fundamental importance for coping with eating problems And nutritional. In addition to these positive points, participants were also mentioned: weight reduction, glycemic and blood pressure control, changes in dietary practices in the family (reduction of salt consumption, sugar, processed foods and increased consumption of salads and fruits), greater care in food preparation, vertical home gardening, greater participation of men in the kitchen, and greater dedication to hiking.

Key words: Food and nutrition education; Primary health care; Group practice. 


\section{INTRODUÇÃO}

As doenças crônicas não transmissíveis (DCNT) configuram um importante problema de saúde pública no Brasil, respondendo pelo grande impacto na morbimortalidade da população. Um grande desafio atual para as equipes de Atenção Básica é a Atenção em Saúde para essas doenças, que são multifatoriais, envolvendo determinantes biológicos e socioculturais. Assim, sua abordagem, para ser efetiva, necessariamente deve envolver as diversas categorias profissionais da equipe de saúde e exigir o protagonismo dos indivíduos, de suas famílias e da comunidade. Nesta perspectiva, torna-se imperiosa a abordagem dos fatores de risco comuns para estes agravos, que incluem o tabagismo, a inatividade física, o consumo de álcool, o excesso de peso e a alimentação inadequada (BRASIL, 2014).

No que se refere à alimentação inadequada, vale salientar que as mudanças não se restringem apenas ao consumo de alimentos, mas sofrem influência de uma constelação de significados ligados ao comer, ao corpo e ao viver (CAISAN, 2014), passando necessariamente pela discussão de questões da Segurança Alimentar e Nutricional (SAN), ainda que no escopo de alimentação/alimento, pois a SAN incorpora aspectos bem mais amplos.

A segurança alimentar e nutricional representa a realização do direito de todas as pessoas ao acesso regular e permanente a alimentos de qualidade, em quantidade suficiente, sem prejudicar o acesso a outras necessidades básicas, tendo como base hábitos alimentares promotoras de saúde, que respeitem a variedade cultural e que sejam social, econômica e ambientalmente sustentáveis (BRASIL, 2006)

Dessa maneira, a questão envolve desde o poder aquisitivo e outros aspectos relacionados à obtenção do alimento até o acesso à informação adequada para a adoção de uma alimentação saudável. Nessa direção, Viana (2002) afirma que escolher uma alimentação saudável não depende apenas do acesso a uma informação nutricional adequada. A seleção de alimentos envolve questões como as preferências desenvolvidas relacionadas com o prazer associado ao sabor dos alimentos, às atitudes aprendidas desde muito cedo na família e a outros fatores psicológicos e sociais. A falta de tempo que caracteriza a sociedade moderna, aliada ao poderoso apelo comercial da indústria alimentícia e seus alimentos prontos para o consumo, favorece a ingestão de alimentos processados e resulta na deterioração dos hábitos tradicionais transmitidos entre gerações.

É necessário, portanto, com o objetivo de tornar mais eficazes as medidas de educação alimentar e nutricional (EAN) para a saúde e de se incorporar hábitos e comportamentos saudáveis na população: 1) compreender o processo de escolhas alimentares do ponto de vista psicológico e sociocultural; e 2) conhecer as atitudes, crenças e outros fatores psicossociais que influenciam este processo de decisão. Assim, a informação nutricional é parte da EAN, de modo que tem sido crescente a sua valorização no contexto da promoção da saúde e da alimentação saudável. A informação nutricional é vista ainda como uma estratégia fundamental para enfrentar os novos desafios nos campos da saúde, alimentação e nutrição (SANTOS, 2012).

EAN significa um conjunto de estratégias sistematizadas para encorajar a cultura e a valorização da alimentação, concebidas no reconhecimento da necessidade de respeitar, mas também ajustar crenças, valores, atitudes, práticas e relações sociais que se estabelecem em torno da alimentação, tendo em vista o acesso econômico e social a uma alimentação adequada em qualidade e quantidade, que atenda aos objetivos de saúde, prazer e convívio social (VASCONCELOS et al., 2008).

Na prática cotidiana de abordagem de portadores de DCNT, percebe-se que a dificuldade dos pacientes em incorporar hábitos alimentares nutricionalmente adequados está em função de diversos fatores, dentre eles o acesso à informação adequada e a falta de tempo e de habilidade no preparo de refeições práticas e saudáveis. É nesse contexto que o presente relato se apresenta, cujo objetivo é descrever uma experiência de trabalho multiprofissional de EAN (o Projeto "Saúde no Prato") para a mudança no estilo de vida de uma amostra de usuários de uma unidade da Atenção Básica, com ênfase na mudança de hábitos alimentares.

\section{MATERIAL E MÉTODOS}

Trata-se de um estudo descritivo do tipo relato de experiência acerca da implantação de um projeto idealizado pela nutricionista da Unidade Básica de Saúde (UBS) e desenvolvido em parceria com residentes de Nutrição, Enfermagem, Serviço Social, Psicologia e Farmácia, do Programa de Residência Multiprofissional em Saúde do Adulto e Idoso do Hospital Universitário Professor Alberto Antunes da Universidade Federal de Alagoas (HUPAA/UFAL) e com profissionais de saúde da UBS, onde é realizado o estágio em Atenção Básica da residência. $\mathrm{O}$ projeto foi realizado nos meses de março a junho de 2016 e se baseia em dois pilares: informação em nutrição e alimentação adequada e oficinas culinárias.

Diante do diagnóstico traçado quanto ao perfil dos usuários da UBS, que incluiu elevado número de pacientes portadores de DCNT e queixas da falta de habilidade e de conhecimento para elaborar preparações saudáveis, das demandas sinalizadas pelos profissionais de saúde e o mais importante, o eixo tratado na publicação do Guia Alimentar para População Brasileira (BRASIL, 2014), a temática "segurança alimentar e nutricional", no escopo relacionado à alimentação e ao alimento (conceitos e preparo de alimentos e alimentação saudável) foi entendida como estratégica e, portanto, escolhida. Diante disso, foi dado curso ao aprofundamento teórico e às discussões sobre a temática entre as residentes, envolvendo particularmente a nutricionista da UBS, que definiram que o projeto teria como base o Guia Alimentar para População Brasileira (BRASIL, 2014).

$\mathrm{Na}$ sequência definiram-se os temas a serem trabalhados e suas estratégias de abordagem, construíramse os recursos pedagógicos e planejou-se a execução. $\mathrm{O}$ formato padrão incluía atividades de grupo com a exposição do tema seguida de uma oficina culinária. Paralelamente foi realizada a divulgação do projeto por meio da distribuição de convites pelos Agentes Comunitários de Saúde durante as visitas domiciliares e 
afixação desses convites, na entrada da UBS, em formato de cartazes.

Foram abertas inscrições para até 25 participantes, estimulando-se a participação de homens e de adolescentes, por se tratar de um público com menor participação nas atividades de grupo da UBS. As inscrições foram realizadas na UBS, mediante preenchimento da ficha de inscrição.

O projeto foi desenvolvido em encontros com os profissionais e usuários inscritos, totalizando seis encontros semanais. $\mathrm{O}$ primeiro encontro foi um momento de acolhimento, com levantamento das expectativas dos participantes e alinhamento destas para o projeto proposto, com a apresentação do "Saúde no Prato" e dos profissionais envolvidos. Nos demais encontros, cada residente trabalhou um tema relacionado à sua área de formação (Tabela 1), e o grupo seguiu com uma oficina culinária que foi conduzida pela nutricionista da UBS. Com o estudo do Guia Alimentar (BRASIL, 2014), constatou-se a pertinência de incluir uma abordagem sobre agrotóxicos e uso de alimentos transgênicos, e ainda sobre atividade física, o que demandou a parceria com um agrônomo e um educador físico.

As oficinas culinárias foram realizadas seguindo a seguinte metodologia: no primeiro encontro os participantes foram questionados sobre as principais dificuldades no preparo dos alimentos, e foram levantadas questões em relação ao interesse por receitas saudáveis que incluíssem ingredientes de baixo custo, que pudessem ser cultivados na própria casa, além de receitas com reaproveitamento de alimentos e de sobremesas de menor conteúdo calórico. Somado a isso foram priorizadas receitas que levassem em suas preparações alimentos in natura ou minimamente processados, que estivessem dentro da realidade dos participantes e relacionadas com o conteúdo apresentado. No último encontro o grupo avaliou a experiência vivenciada, por meio de um questionário, com a exposição dos pontos positivos e dos que precisavam ser trabalhados.

Tabela 1. Temas abordados no projeto "Saúde no Prato" dentro da temática central Segurança Alimentar e Nutricional (SAN).

\begin{tabular}{lcc}
\hline $\begin{array}{l}\text { Profissional } \\
\text { responsável }\end{array}$ & Tema & Objetivo \\
\hline
\end{tabular}

Nutricionistas Alimentação saudável e tipos de alimentos segundo processamento (Alimentos in natura, processados e ultraprocessados).

Assistentes Sociais

Alimentação adequada e saudável: direito de todos;

Direito humano à alimentação adequada.

Enfermeiras

Psicólogos Transtornos alimentares.
Discutir sobre a transição alimentar e nutricional, os tipos de alimentos (Alimentos in natura, processados e ultraprocessados) e as escolhas mais adequadas para uma alimentação saudável.

Identificar a alimentação como um direito social e descrever os diferentes modelos de produção agrícola (agronegócio e agricultura familiar).

Descrever os cuidados na manipulação dos alimentos desde a aquisição, manipulação e armazenamento, até o seu consumo.

Identificar transtornos ligados à alimentação, como por exemplo, a apelação psicológica da mídia, disposição estratégica de prateleiras em supermercados, compulsão alimentar e até transtornos mais graves.
Farmacêuticos Alimentação segura: uso de plantas medicinais e alimentos funcionais.

Agrônomo

Educador físico Alimentação segura: alimentos transgênicos
e agrotóxicos.
Descrever propriedades terapêuticas de plantas medicinais e alimentos funcionais.

Relacionar os malefícios para a saúde no consumo de alimentos transgênicos e propor alternativas para o consumo de alimentos mais livres de agrotóxicos.

Orientar práticas seguras de caminhadas e outras atividades físicas.

\section{RESULTADOS E DISCUSSÃO}

\section{Histórico, equipe executora e objetivos do projeto}

O Projeto "Saúde no Prato" teve inicio em 2016, vinculado ao Programa de Residência Multiprofissional em Saúde do Adulto e Idoso do Hospital Universitário Professor Alberto Antunes da Universidade Federal de Alagoas (HUPAA/UFAL) e executado na UBS do Graciliano Ramos, bairro circunvizinho do HUPAA/UFAL, durante a vivência na prática em Saúde Coletiva das residentes. O Projeto foi idealizado pela nutricionista da UBS a partir de inquietações durante sua vivência, que apontaram uma necessidade de intervenção em amplitude coletiva e de caráter multiprofissional.

Tratou-se de uma ação de promoção da saúde, voltada para a população de abrangência da UBS Graciliano Ramos, realizada na sede da Associação de Moradores do bairro, escolhido estrategicamente por situar-se próximo à UBS e por dispor de uma cozinha para a execução das oficinas. A ação aconteceu em reuniões semanais cujo conteúdo contemplou uma explanação teórica e um momento prático de oficina culinária.

A denominação do projeto foi apresentada pela nutricionista da UBS e obteve total aceitação por parte dos 
residentes, que consideravam necessárias uma abordagem de grupo e multiprofissional de sensibilização para mudança de hábitos alimentares na prevenção e tratamento das DCNT, como uma ação de promoção da saúde. Daí a definição de "Saúde" (que remete à melhora na saúde como um todo) "no Prato" (sugerindo começar a mudança nas escolhas alimentares).

No que diz respeito ao trabalho em equipe, os objetivos do projeto foram: (1) contribuir para a disseminação deste modelo de intervenção na Atenção Básica; (2) estimular e evidenciar a importância da atuação multiprofissional na Atenção Básica; e (3) fomentar a troca de experiência entre residentes, profissionais do serviço e a comunidade. Já no que diz respeito à população-alvo, enquanto ação de EAN per si, seu objetivo foi promover mudança no estilo de vida em uma amostra de usuários de uma unidade da Atenção Básica, com ênfase na mudança de hábitos alimentares.

\section{População-alvo}

A população-alvo foi composta por usuários e profissionais da UBS. Devido à grande procura, participaram 40 pessoas, com idades entre 19 e 75 anos de idade e a maioria dos participantes apresentava algum tipo de DCNT, predominando a hipertensão arterial. Um número elevado de participantes apresentava ainda excesso de peso. Os profissionais que participaram eram desde assistentes administrativos até profissionais de saúde, como médica, dentista, enfermeiras e agentes comunitários de saúde.

As DCNT constituem um grande problema de Saúde Pública na atualidade. Segundo dados da Organização Mundial da Saúde (OMS), essas doenças são responsáveis por aproximadamente $80 \%$ das mortes em países de baixa e média renda. $\mathrm{O}$ excesso de peso e a distribuição da gordura corporal associam-se fortemente com maior risco para doenças crônicas, como a hipertensão arterial e o diabetes mellitus. Em relação à magnitude epidemiológica, estudos mostram tendência crescente do excesso de peso e obesidade abdominal nos países desenvolvidos e em desenvolvimento, assim como o presente estudo mostra elevado número de portadores de DCNT e excesso de peso. No Brasil, dados da Pesquisa de Orçamentos Familiares (POF) 2008-2009 evidenciam aumento do excesso de peso em adultos desde a década de 1970. No estudo de Cristóvão et al. (2011), que avaliou o excesso de peso e obesidade abdominal em mulheres atendidas em Unidade de Saúde da Família foi constatado que o excesso de peso atingia 56\% das participantes, sendo que destas $36,9 \%$ tinham sobrepeso e $19,1 \%$ obesidade.

Corroborando com os dados encontrados no presente estudo, o trabalho de Hollo, Leite e Navarro (2007), que teve como objetivo destacar a importância da educação alimentar na prática de adoção de hábitos alimentares saudáveis, de mulheres atendidas em Unidade Básica de Saúde (UBS), mostrou que todas as mulheres apresentavam algum grau de excesso de peso: $14 \%$ sobrepeso e $86 \%$ obesidade, sendo $32 \%$ com obesidade grau I (IMC 30 a 34,9 kg/m²), 50\% com obesidade grau II (35 a $39,9 \mathrm{~kg} / \mathrm{m}^{2}$ ) e $3,5 \%$ com obesidade grau III (IMC > $\left.40 \mathrm{~kg} / \mathrm{m}^{2}\right)$.

\section{Processo de execução}

Nos encontros iniciais, questionou-se junto aos participantes sobre as motivações que os trazia ao grupo. A maioria relatou suas condições de excesso de peso, falta de habilidade para preparações culinárias mais saudáveis, falta de conhecimento sobre alimentação saudável, além da intenção de buscar uma solução para os seus problemas de saúde, condição que os limitava na execução de tarefas rotineiras e comprometia o convívio familiar. Evidenciaram-se, por vezes, em algumas falas, condutas passivas em relação aos próprios problemas, em que as pessoas apenas descreviam sua condição de saúde e o quanto essa se encontrava prejudicada. Um depoimento foi além, com a declaração de que a condição da saúde e bem-estar passou a configurar uma preocupação, substituindo o incômodo anteriormente restrito à forma física. Para a maioria das pessoas, no entanto, tratava-se de uma dinâmica diferente, que saía da rotina de consultas tradicionais, sendo este o ponto positivo mais citado.

A ação educativa na atenção primária estabelece-se a partir de programas determinados verticalmente, ou de forma conjunta às ações de promoção da saúde e prevenção da doença junto à comunidade, indivíduos ou grupos sociais, permeando densamente as atividades que os profissionais de saúde realizam no âmbito das unidades, no domicílio, em outras instituições e nos espaços comunitários. Bons resultados nesse campo vão contribuir para diminuir a procura dos usuários pela unidade de saúde, proporcionando-lhe maior satisfação com seu autocuidado (DIAS et al., 2009).

Ainda, nos encontros iniciais foram marcantes os momentos de descontração e interação entre os participantes, o que favoreceu a adesão e participação ativa dos integrantes nas temáticas desenvolvidas ao longo do processo. O trabalho de grupos em atenção primária é uma alternativa para as práticas assistenciais. Estes espaços favorecem o aprimoramento de todos os envolvidos, não apenas no aspecto pessoal como também no profissional, por meio da valorização dos diversos saberes e da possibilidade de intervir criativamente no processo de saúde-doença de cada pessoa.

Os temas das palestras foram distribuídos entre as residentes e os respectivos profissionais levando em consideração o que era de competência de cada área e tendo como tema central a segurança alimentar e nutricional (SAN). Embora a assistência e educação nutricional constituam ações privativas do nutricionista, conforme disposto na Lei 8.234/91 (BRASIL, 1991), que regulamenta a sua atuação profissional, a promoção da alimentação saudável tem caráter mais amplo, perpassando não só ações de outros profissionais, como também iniciativas que transcendem os serviços de saúde (BRASIL, 2012; BOOG, 2008).

Com o estudo do Guia Alimentar (BRASIL, 2014), constatou-se a pertinência de incluir uma abordagem sobre agrotóxicos e uso de alimentos transgênicos, o que demandou a parceria com um agrônomo, que sugeriu oficinas para preparação de horta vertical como estratégia para discutir o assunto e sensibilizar a população quanto ao consumo de alimentos transgênicos. Nas sociedades modernas a indústria alimentícia tem centralidade crescente na vida cotidiana. $\mathrm{O}$ alimento em estado natural, fresco, vindo direto do agricultor foi, aos poucos, 
substituído por alimentos com graus cada vez maiores de processamento, artificializando-se, embora muitas vezes lhe seja atribuída a denominação de "natural" para manter a necessária conexão com as expectativas do consumidor. Assim, a ingestão de alimentos industrializados e alimentos modificados geneticamente por meio da biotecnologia pode ser considerada um risco em potencial à saúde (GALINDO; PORTILHO, 2015), haja vista o seu conteúdo de aditivos e substancias que comprometem o seu valor nutricional.

As atividades realizadas no projeto incluíram debates entre os participantes e a equipe executora sobre os temas estabelecidos, além da utilização de metodologias ativas, como dinâmicas, jogos e trocas de experiências, o que despertava o interesse dos participantes, como foi possível observar em falas como "com essa dinâmica é muito mais interessante e mais fácil de aprender".

Segundo Miltre et al. (2008), o grande desafio deste início de século está na perspectiva de se desenvolver a autonomia individual em íntima coalizão com o coletivo. A educação deve ser capaz de desencadear uma visão do todo de interdependência e de transdisciplinaridade, além de possibilitar a construção de redes de mudanças sociais, com a consequente expansão da consciência individual e coletiva. Portanto, um dos seus méritos está, justamente, na crescente tendência de buscar métodos inovadores, que admitam uma prática pedagógica ética, crítica, reflexiva e transformadora, ultrapassando os limites do treinamento puramente técnico para efetivamente alcançar a formação do homem como um ser histórico, inscrito na dialética da ação-reflexão.

As oficinas culinárias foram realizadas com participação ativa de todos, seguida de degustação. Foram escolhidas receitas que além de saudáveis eram simples, rápidas e de baixo custo, utilizando alimentos/preparações pouco ou até não conhecidos (semente de girassol, tabule), de pouca aceitação (arroz integral) e conhecidos, mas pouco consumidos (saladas), cujas propriedades e conteúdo nutricional, apresentados nas oficinas, agregariam valor à dieta dos participantes, considerando o seu perfil (Tabela 2). Dicas de reutilização de alimentos e de manipulação que preservasse ao máximo os nutrientes, foi o que grande parte dos participantes relatou ter aprendido nas oficinas. No primeiro encontro, a preparação escolhida, o arroz integral, com receitas de preparo para dar maior palatabilidade, levou em consideração as principais queixas dos participantes durante as consultas com a nutricionista e o fato de ser uma preparação de consumo diário no cotidiano das pessoas.

No último encontro, o educador físico do Núcleo de Apoio à Saúde da Família (NASF) abordou ainda a importância da atividade física e os riscos do sedentarismo para a saúde. É possível assumir que as UBS, presentes em praticamente todos os municípios brasileiros, seriam locais privilegiados para a implantação de ações de promoção da saúde envolvendo exercício físico, tendo em vista que atenderiam parte da população que não possui acesso a programas para a prática de exercício físico de qualidade ou que pertencem a minorias altamente suscetíveis a um estilo de vida inativo. Neste contexto, discutiu-se a inserção das ações do NASF nas UBS como uma medida que deve ser incentivada. Importantes estudos têm demonstrado os inúmeros benefícios da prática de atividade física (GONÇALVES et al., 2011), seja no controle de peso e nas alterações metabólicas para o controle de DCNT, seja nos aspectos, psicológicos e sociais dos seus praticantes, especialmente quando adotado com regularidade e sob orientação de profissional da área.

Ao final de todos os encontros foi disponibilizado material educativo com os principais pontos abordados, além das receitas que foram trabalhadas nas oficinas. Neste momento ainda, os participantes foram questionados se foram alcançadas as expectativas, além de serem encorajados a apontar sugestões, pontos positivos e pontos negativos da experiência vivenciada. Foi unânime a sugestão de aumentar o tempo das sessões e o número de encontros. Para tal, foram sugeridas outras edições, com outros temas e novas receitas.

Foi possível observar o sucesso do projeto ao longo dos encontros, pois as pessoas participaram de forma ativa, trazendo um feedback positivo. Foram citados pelos participantes os seguintes pontos positivos: redução do peso e da pressão arterial, mudanças de práticas alimentares na família (como, por exemplo, redução do consumo de sal, açúcar e alimentos industrializados e aumento no consumo de saladas e frutas), maior cuidado na higiene dos alimentos, confecção de horta vertical em casa, maior participação dos homens na cozinha (uma vez que as mulheres que participaram acabaram sendo multiplicadoras, levando com elas o incentivo para a participação de seus companheiros), e uma maior 'dedicação' às caminhadas.

No estudo de Silva et al. (2006) de intervenção com grupos de pacientes, foi utilizado um modelo de educação interativa, segundo o qual os pacientes eram estimulados a identificar suas necessidades e dificuldades, refletindo sobre as situações cotidianas. A troca de vivências e experiências entre os membros do grupo foi estimulada para adequar a essa população específica os conhecimentos necessários sobre doença, seus determinantes e seu controle.

No estudo de Cunha e Albano (2012), que se caracteriza como série de casos envolvendo mulheres adultas que participaram de atividades de educação nutricional em grupo, durante 12 semanas, realizada em um ambulatório-escola de uma Instituição de Ensino Superior, localizada no município de Santos-SP, foi possível observar que após a intervenção de 12 semanas, houve reduções estatisticamente significantes de todos os parâmetros analisados (IMC, circunferência abdominal, percentual de gordura, massas magra e gorda), para as adultas. Para o grupo de idosas, observaram-se diferenças significantes em redução do valor médio de IMC, massa gorda e magra em quilos. 
Tabela 2. Preparações selecionadas para as oficinas culinárias do Projeto "Saúde no Prato".

\begin{tabular}{|c|c|c|c|}
\hline & PREPARAÇÕES & MOTIVO DA ESCOLHA & OBJETIVO \\
\hline $\begin{array}{l}\text { Primeiro } \\
\text { encontro }\end{array}$ & $\begin{array}{l}\text { Arroz integral } \\
\text { O arroz integral é considerado o tipo de arroz } \\
\text { mais saudável por seu alto teor de fibras e baixo } \\
\text { índice glicêmico, além da grande quantidade de } \\
\text { vitaminas e minerais. }\end{array}$ & $\begin{array}{lccr}\text { Relatos } & \text { sobre } & \text { a baixa } \\
\text { aceitação pelos } & \text { participantes } \\
\text { devido ao } & \text { sabor } & \text { e } \\
\text { consistência. } & & \end{array}$ & $\begin{array}{l}\text { - Mostrar formas para preparar o } \\
\text { arroz integral mais saboroso. } \\
\text { - Estimular o consumo de arroz } \\
\text { integral em substituição ao arroz } \\
\text { polido. }\end{array}$ \\
\hline
\end{tabular}

Segundo Girassol crocante

encontro A semente de girassol é considerada um alimento.

É rica em proteínas, vitaminas e minerais e pode ser facilmente consumido nos lanches, além de ser de fácil acesso.

Iogurte natural

O iogurte é um alimento muito nutritivo e de melhor aceitação que o leite. Assim como o leite, é fonte de cálcio e potássio, fósforo, vitaminas $\mathrm{A}$, $\mathrm{B}_{6}$ e $\mathrm{B}_{12}$, riboflavina, ácido fólico e niacina. Possui mais ácido fólico do que o leite, por conta da atividade das bactérias envolvidas na fermentação.

Terceiro Salada colorida maravilhosa

encontro A salada de verdura e legumes consiste em uma reparação fundamental nas grandes refeições de uma alimentação saudável. Além do baixo valor calórico, contém alimentos ricos em vitaminas, minerais e fibras.

Molho de iogurte para salada

$\mathrm{O}$ uso de molho para saladas à base de iogurte constitui uma estratégia saudável para agregar nutrientes além de conferir sabor ao prato. Fonte de cálcio e potássio, fósforo, vitaminas $A, B_{6}$ e $\mathrm{B}_{12}$, riboflavina, ácido fólico e niacina.

Quarto Salada de frutas sem adição de açúcar

encontro $\mathrm{O}$ consumo diário de frutas e em variedade deve ser estimulado para estender aos princípios de uma alimentação saudável. As frutas são fontes de vitaminas, minerais e fibras, além de possuírem ação antioxidante.

Creme de coco dos deuses

Sobremesa com menor valor calórico em comparação à convencional, além de oferecer vitaminas e minerais que fortalecem o sistema imunológico.

Quinto Tabule

encontro $\mathrm{O}$ tabule é conhecido também como salada árabe, reúne uma grande quantidade de ingredientes saudáveis: o trigo para quibe que é rico em vitaminas do complexo B, fonte de fibras, potássio, fósforo, cálcio, magnésio, selênio, manganês e ferro; e, as verduras tomate, cebola, pimentão, pepino, repolho e alface, que são fontes de vitaminas, minerais e fibras.

Seiva da terra (ou suco verde)

O suco verde é feito com vegetais de folha verde escura, frutas, legumes e grãos de fácil acesso e é capaz de reunir diversos nutrientes importantes para o corpo, como ferro, vitamina $\mathrm{C}$, ácido fólico e magnésio, além de fibras e antioxidantes.
Pouco ou não conhecimento dos participantes da semente de girassol como alimento para consumo humano.

Baixa aceitação ou hábito por parte dos participantes do leite para o consumo diário.

Baixo consumo pelos
participantes devido ao pouco participantes devido ao pouco
conhecimento sobre a variedade de hortaliças disponíveis para salada.

Alternativa para aumentar o consumo de saladas e melhorar a sua aceitação pelos participantes.

Monotonia nas escolhas das - Incentivar o consumo de frutas frutas por parte dos variadas. participantes.

Pouco ou não conhecimento dos participantes sobre alternativas saudáveis de sobremesas.

Baixo consumo pelos - Incentivar o consumo de saladas participantes devido ao pouco variadas.

conhecimento sobre a variedade de legumes, hortaliças e verduras disponíveis para salada.

Pouco ou não conhecimento - Incentivar o consumo de sucos dos participantes sobre naturais a partir da junção de variedades naturais de suco. vários tipos de frutas e vegetais.
A educação nutricional constitui uma estratégia de fundamental importância para o enfrentamento dos problemas alimentares e nutricionais encontrados na atualidade. Não se concebe mais a prática da educação nutricional que consista apenas na transmissão de conceitos de nutrição para a população, com palestras 
cujos conteúdos limitem-se aos aspectos biológicos da alimentação, destacando o efeito dos nutrientes no organismo. Embora esta seja uma informação importante, não considera os aspectos regionais e as desigualdades sociais que comprometem o acesso da população a uma alimentação adequada, afetando a eficiência da educação nutricional (RODRIGUES; RONCADA, 2008). É fundamental que as ações de EAN sejam contextualizadas e materializadas com base nos interesses do público-alvo.

Neste sentido, Rebert et al. (2012) discutem que os modelos de comunicação, baseados na relação dialógica e em princípios multidirecionais, permitem a existência do diálogo entre as pessoas envolvidas no processo de construção de ações. A interação e a troca de conhecimentos, considerando-se o estilo de vida das pessoas, são aspectos essenciais nesse processo. A adoção de uma abordagem participativa, comunicativa e coletiva é recomendada nas ações educativas. A educação em saúde é uma prática social, devendo ser centrada na problematização do cotidiano, na valorização da experiência de indivíduos e grupos sociais e na leitura das diferentes realidades. Vista como prática social, a educação em saúde passou a ser repensada como um processo capaz de desenvolver a reflexão e a consciência crítica das pessoas sobre as causas de seus problemas de saúde, enfatizando o desencadeamento de um processo baseado no diálogo, de modo que se passe a trabalhar com as pessoas e não mais para as pessoas. Esse diálogo assume o pressuposto de que todos possuem potencial para serem protagonistas de sua própria história, de que estão motivados para se organizarem e de que possuem expectativas sobre as possibilidades de mudança (ALVES; AERTS, 2011).

Diante da avaliação, as novas edições do "Saúde no Prato" estão sendo planejadas para atingir o máximo de pessoas da comunidade, diversificando o público e aumentando a frequência de turmas. A partir de vivências como esta, estabelece-se um espaço de reflexão para desenvolver novas estratégias de atenção em saúde de modo a promover motivação com autonomia. $\mathrm{O}$ paciente torna-se, assim, capaz de identificar barreiras para o autocontrole e desenvolver mecanismos para superá-las. O indivíduo deverá prevenir recaídas, fazer melhores escolhas diante dos desafios cotidianos e adquirir a habilidade para resolver problemas e, portanto, obter melhores resultados no tratamento (GUIMARÃES et al., 2010). Neste sentido, é de fundamental importância a atuação multiprofissional, fortalecendo a premissa de que o indivíduo precisa ser visto de forma global. A ação multiprofissional conjunta e articulada é mais eficaz para promover mudanças nos hábitos de vida, o que foi constatado nesta experiência com respeito às escolhas alimentares.

De acordo com Leal et al. (2015), no campo da saúde coletiva, algumas reflexões têm sido produzidas acerca do trabalho em equipe, como forma de modificar o modelo de atenção à saúde. A comunicação, portanto, é considerada o denominador comum do trabalho conjunto. Experiências de ações educativas em equipe reafirmam que, quando há comunicação, há interação no trabalho coletivo e menor desigualdade entre os diferentes trabalhos e os respectivos agentes. Por conseguinte, ocorre maior integração e melhores são os resultados.

\section{CONCLUSÕES}

Com o multiprofissionalismo o trabalho em equipe, ganha uma nova dimensão no sentido da divisão de responsabilidades do cuidado entre os diversos membros da equipe, na qual todos participam com suas especificidades contribuindo para a qualidade da prestação das ações de saúde. A concepção integral do cuidado favorece a ação interdisciplinar nas práticas, em que a valorização das diversas disciplinas contribui para uma abordagem ampla e resolutiva do cuidado. A importância do diálogo na busca do consenso constitui elemento imprescindível para o bom desenvolvimento do trabalho em equipe. Isso aponta para a possibilidade do desenvolvimento de uma prática comunicativa. O trabalho conjunto "provoca" a escuta do outro, o que pressupõe o estabelecimento de um canal de comunicação.

Para a população-alvo, as ações realizadas apresentam grande importância para as medidas de saúde coletiva, pois tendem a exercer significados de bem-estar oportunos aos participantes, colaborando na reflexão e no aprendizado de como proceder diante de situações de vida que influenciam no processo saúde-doença.

\section{REFERÊNCIAS}

ALVES, G. G.; AERTS, D. As práticas educativas em saúde e a Estratégia Saúde da Família. Ciência \& Saúde Coletiva, v. 16, n.1, p. 319-325, 2011.

BELIK, W. Perspectivas para segurança alimentar e nutricional no Brasil. Saúde e Sociedade, v.12, n.1, p.1220, 2003.

BOOG, M. C. F. Atuação do nutricionista em saúde pública na promoção da alimentação saudável. Revista Ciência \& Saúde, Porto Alegre, v. 1, n. 1, p. 33-42, 2008.

BRASIL. Lei $\mathrm{n}^{\circ} 8.234$ de 17 de Setembro de 1991. Regulamenta a profissão de Nutricionista e determina outras providências. Diário Oficial da União, 1991.

BRASIL. Lei ${ }^{\circ}$ 11.346, de 15 de setembro de 2006. Cria o Sistema Nacional de Segurança Alimentar e Nutricional - SISAN com vistas em assegurar o direito humano à alimentação adequada e dá outras providências. Diário Oficial da União, 2006

BRASIL. Ministério da Saúde. Secretaria de Atenção à Saúde. Departamento de Atenção Básica. Estratégias para o cuidado da pessoa com doença crônica. Brasília: MS, 2014.

BRASIL. Ministério do Desenvolvimento Social e Combate à Fome. Marco de referência de educação alimentar e nutricional para as políticas públicas. Brasília, DF, 2012.

BRASIL. Ministério da Saúde. Secretaria de Atenção à Saúde. Departamento de Atenção Básica. Guia alimentar para a população brasileira. 2. ed. Brasília: 2014.

CAISAN. Câmara Interministerial de Segurança Alimentar e Nutricional Estratégia Intersetorial de Prevenção e Controle da Obesidade: recomendações para estados e municípios. Brasília, DF, 2014.

CHAVES, D. F. S. Compostos bioativos dos alimentos, 
coleção nutrição clínica funcional. São Paulo: VP, 2015. 338p. 2015.

CRISTÓVÃO, M. F.; SATO, A. P. S.; FUJIMORI, E. Excesso de peso e obesidade abdominal em mulheres atendidas em Unidade da Estratégia Saúde da Família. Rev Esc Enferm, v. 45, p. 1667-72, 2011.

CUNHA, D. T.; ALBANO, R. D. Educação nutricional por meio de atividade de grupo: o desempenho de intervenção educativa de curto prazo. Rev Bras Nutr Clin, v. 27, n.3, p. 170-5, 2012.

DIAS, V. P.; SILVEIRA, D. T.; WITT, R. R. Educação em saúde: o trabalho de grupos em atenção primária. Rev. APS, v. 12, n. 2, p. 221-227, 2009.

GALINDO, F.; PORTILHO, F. "O Peixe Morre pela Boca": Como os Consumidores Entendem os Riscos dos Agrotóxicos e dos Transgênicos na Alimentação. Sustentabilidade em Debate. Brasília, v. 6, n. 2, p. 73-87, 2015.

GUIMARÃES, N. G.; DUTRA, E. S.; ITO, M. K.; CARVALHO, K. M. B. Adesão a um programa de aconselhamento nutricional para adultos com excesso de peso e comorbidades. Rev. Nutr., Campinas, v. 23, n. 3, p. 323-333, 2010.

GONÇALVES, A. K. S.; CANÁRIO, A. C. G.; CABRAL, P. U. L.; SILVA, R. A. H.; SPYRIDES, M. H. C.; GIRALDO, P. C.; JR. E. J. Impacto da atividade física na qualidade de vida de mulheres de meia idade: estudo de base populacional. Rev Bras Ginecol Obstet, v. 33, n. 12. p. 408-13, 2011.

HOLLO, R. A. M.; LEITE, M. D. O.; NAVARRO, F. A educação nutricional como forma de viabilizar o tratamento de mulheres com sobrepeso e obesidade, com baixa renda, atendidas numa Unidade Básica de Saúde (UBS), no município de Cabreúva, SP. Revista Bras de Obes, Nut e Emag, São Paulo v.1, n. 4, p.109-118, 2007.

KALLUF, L. Fitoterapia Funcional: dos princípios ativos à prescrição de fitoterápicos. 2.ed. São Paulo: AçãoSet, 2015. $346 \mathrm{p}$.

KOKUBUN, E.; LUCIANO, E.; SIBUYA, C. Y.; QUEIROGA, M. R.; RIBEIRO, P. A. B.; SILVEIRA, R. F.; NAKAMURA, P. M. Programa de atividade física em unidades básicas de saúde: relato de experiência no município de Rio Claro-SP. Revista Brasileira de Atividade Física \& Saúde, 2007.

LEAL, J. A. L.; MELO, C. M. M.; VELOSO, R. B. P; JULIANO, I. A. Novos espaços de reorientação para formação em saúde: vivências de estudantes. Comunicação Saúde Educação, v. 19, n. 53, p. 361-71, 2015.

MAHAM, L. K.; ESCOTT-STUMP, S. Krause: Alimentos, nutrição e dietoterapia. 13.ed. Rio de Janeiro: Elsevier, 2012. 1227 p.

MAFFACCIOLLI, R.; LOPES, M. J. M. Educação em Saúde: a orientação alimentar através de atividades de grupo. Acta Paul Enferm, v. 18, n. 4, p. 439-45, 2005.
MILTRE, S. M. BATISTA, R. S.; MENDONÇA, J. M. G.; PINTO, N. M. M.; MEIRELLES, C. A. B.; PORTO, C. P.; MOREIRA, T.; HOFFMANN, L. M. A. Metodologias ativas de ensino-aprendizagem na formação profissional em saúde: debates atuais. Ciência \& Saúde Coletiva, v.13, p. 2133-2144, 2008.

MORETTI, A. C.; ALMEIDA, V.; WESTPHAL, M. F.; BÓGUS, C. M. Práticas Corporais/Atividade Física e Políticas Públicas de Promoção da Saúde. Saúde Soc, v.18, n.2, p.346-354, 2009.

OLIVEIRA, L. L.; HUTZ, C.S. Transtornos alimentares: o papel dos aspectos culturais no mundo contemporâneo. Psicologia em Estudo, Maringá, v. 15, n. 3, p. 575-582, 2010.

PRIMO, M. G. B.; RIBEIRO, L. C. M.; FIGUEIREDO, L. F. S.; SIRICO, S. C. A.; SOUZA, M. A. Adesão à prática de higienização das mãos por profissionais de saúde de um Hospital Universitário. Rev. Eletr. Enf, v. 12, n. 2, p. 266-71. 2010.

ROBERTE, L. M.; HOGA, L. A. K.; GOMES, A. L. Z. O processo de construção de material educativo para a promoção da saúde da gestante. Rev. Latino-Am. Enfermagem, v. 20, n. 1, 2012.

RODRIGUES, L. P. F.; RONCADA, M. J. Educação nutricional no Brasil: evolução e descrição de proposta metodológica para escolas. Ciências Saúde, v. 19, n. 4, p. 315-322, 2008

ROSSI, A.; MOREIRA, E. A. M.; RAUEN, M. S. Determinantes do comportamento alimentar: uma revisão com enfoque na família. Rev. Nutr., Campinas, v. 21, n. 6 , p. 739-748, 2008.

SANTOS, L. A. S. O fazer educação alimentar e nutricional: algumas contribuições para reflexão. Ciência \& Saúde Coletiva, v. 17, n. 2, p. 453-462, 2012.

SILVA, T. R; FELDMAM, C.; LIMA, M. H. A.; NOBRE, M. R. C.; DOMINGUES, R. Z. L. Controle de Diabetes Mellitus e Hipertensão Arterial com Grupos de Intervenção Educacional e Terapêutica em Seguimento Ambulatorial de uma Unidade Básica de Saúde. Saúde e Sociedade, v.15, n.3, p.180-189, 2006.

VASCONCELOS, A. C. C. P.; PEREIRA, I. D. F.; CRUZ, P. J. S. C. Práticas educativas em nutrição na Atenção Básica em Saúde: reflexões a partir de uma experiência de extensão popular em João Pessoa-Paraíba. Rev. APS, v. 11, n. 3, p. 334-340, 2008.

VIANA, V. Psicologia, saúde e nutrição: Contributo para o estudo do comportamento alimentar. Análise Psicológica, n. 4, p. 611-624, 2002.

WHO. World Heath Organization. Global status report on noncommunicable diseases 2010: description of the global burden of NCDs, their risk factors and determinants. Geneva: World Heath Organization; 2011.

WHO. World Health Organization. Physical Status: The Use and Interpretation of Anthropometry. Geneva: World Health Organization, 1995. 